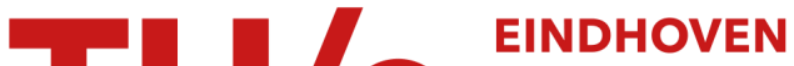 UNIVERSITY OF TECHNOLOGY
}

\section{Photonic integration technologies for indoor optical wireless communications}

Citation for published version (APA):

Jiao, Y., \& Cao, Z. (2018). Photonic integration technologies for indoor optical wireless communications. SCIENCE CHINA Information Sciences, 61(8), [080404]. https://doi.org/10.1007/s11432-018-9391-1

DOI:

10.1007/s11432-018-9391-1

Document status and date:

Published: 01/08/2018

Document Version:

Accepted manuscript including changes made at the peer-review stage

Please check the document version of this publication:

- A submitted manuscript is the version of the article upon submission and before peer-review. There can be important differences between the submitted version and the official published version of record. People interested in the research are advised to contact the author for the final version of the publication, or visit the $\mathrm{DOI}$ to the publisher's website.

- The final author version and the galley proof are versions of the publication after peer review.

- The final published version features the final layout of the paper including the volume, issue and page numbers.

Link to publication

\section{General rights}

Copyright and moral rights for the publications made accessible in the public portal are retained by the authors and/or other copyright owners and it is a condition of accessing publications that users recognise and abide by the legal requirements associated with these rights.

- Users may download and print one copy of any publication from the public portal for the purpose of private study or research.

- You may not further distribute the material or use it for any profit-making activity or commercial gain

- You may freely distribute the URL identifying the publication in the public portal.

If the publication is distributed under the terms of Article $25 \mathrm{fa}$ of the Dutch Copyright Act, indicated by the "Taverne" license above, please follow below link for the End User Agreement:

www.tue.nl/taverne

Take down policy

If you believe that this document breaches copyright please contact us at:

openaccess@tue.nl

providing details and we will investigate your claim. 


\section{SCIENCE CHINA \\ Information Sciences}

\section{Photonic Integration Technologies for Indoor Optical Wireless Communications}

ఏiao Yuqing and Cao Zizheng

Citation: SCIENCE CHINA Information Sciences ; doi: 10.1007/s11432-018-9391-1

View online: http://engine.scichina.com/doi/10.1007/s11432-018-9391-1

Published by the Science China Press

\section{Articles you may be interested in}

Integrated photonic orbital angular momentum devices and systems: Potentials and challenges

SCIENCE CHINA Technological Sciences 56, 579 (2013);

Silicon photonic network-on-chip and enabling components

SCIENCE CHINA Technological Sciences 56, 543 (2013);

Photonic integrated technology for multi-wavelength laser emission

Chinese Science Bulletin 56, 3064 (2011);

Development of silicon photonic devices for optical interconnects

SCIENCE CHINA Technological Sciences 56, 586 (2013);

Data-aided synchronization algorithm dispensing with searching procedures for UWB communications SCIENCE CHINA Information Sciences 55, 778 (2012); 


\section{Introduction}

Abstract Indoor optical wireless communication (OWC) using steerable infrared beams is regarded as an important component in future 5G network. Photonic integration technologies can meet the criteria of such application, and provide low-cost, high-performance and very compact chips. In this paper we review the recent development of photonic integration technologies suitable for indoor OWC application, and discuss in detail the current status and future opportunities of several key devices, such as the chip to free space couplers, integrated receivers and transmitters.

Keywords Photonic Integration, indium phosphide (InP), silicon, 5G, optical wireless communication (OWC)

Citation Jiao Y, et al. Photonic Integration Technologies for Indoor Optical Wireless Communications. Sci China Inf Sci, for review

Connecting and communicating with multitude of physical devices using wireless technology is an important part of the internet of things (IoT). It was predicted that by year of 2020, there will be 6-7 devices connected per person [1]. Significant part of these devices will be connected wirelessly. This requires a revolutionary upgrade in the data capacity of the current wireless network, in order to support the ultra-high capacity data transmission from and to the device cluster as well as the data exchange among the devices. The next-generation (5G) wireless network is demanded to facilitate a 1000 times boost in capacity [2] and support the rapidly development of IoT.

The indoor home network is one of the most important scenario of the future $5 \mathrm{G}$ network [3]. The number of devices connected wirelessly at home area is exploding in recent years. Besides computers and smartphones, smart house appliances with integrated sensors, actuators and communication units are becoming key elements for smart home applications [4]. Those devices at home need to exchange data with each other on a real-time basis. Through an access point in every room, the devices in different rooms can then be connected. Another important application in the home area is the healthcare. Health conditions of the family members are monitored through various sensors and monitors. This can include regular tracking of exercises of adult family members, as well as real-time monitoring of elders and children. Such a healthcare network can be constructed using the framework of IoT and cloud computing technology [5]. Similarly, the home network technology can be expanded to industrial applications such as the factory of the future.

\footnotetext{
*Corresponding author (email: y.jiao@tue.nl)
} 
Jiao Y, et al. Sci China Inf Sci 2

Ultra-high capacity data communication is envisaged for such smart home networks [3]. One way to accommodate the explosion of data capacity is to move the radio frequency used in the current wireless network towards higher frequencies, such as the microwave and millimeter-wave bands [6]. Another substantial advancement in recent years is the optical wireless communication (OWC) technology [7]. The optical technology works with much wider spectral resources in a much higher carrier frequency, compared with the radio technology. Therefore the OWC technology is regarded as a promising solution for the huge data exchange in IoT applications [8]. Visible light communication (VLC) using existing indoor lighting system (e.g., the LEDs) has been studied intensively in recent years $[9,10]$. This technology is omni-directional, which has disadvantages such as capacity-sharing by multiple terminals, severe power attenuation at distance and insecurity [7]. Another approach, based on focused and steerable infrared beams (1.55 $\mu \mathrm{m}$ telecom wavelength), is gaining more and more attention due to the advantages in capacity and security. Tens of Gbit/s data can be transmitted and received using a focused infrared beam $[7,11]$, which is one to two orders of magnitude advancement as compared with typical VLC [9]. Therefore the infrared OWC technology is a promising candidate for the future ultra-high capacity indoor communications.

Another critical requirement for the smart home network is the cost. As a large number of smart devices to be deployed and connected, the optical wireless devices have to be sufficiently low cost. Therefore photonic integration is highly demanded in the indoor OWC application for its compactness, high performance and more importantly mass manufacturability and reduced cost. The maturity of photonic integration has evolved significantly in recent years. Nearly 2000 functional components have been integrated in a single chip [12]. Such high density and complexity are suitable for realization of fully integrated optical wireless transmitters and receivers. The success of generic photonic integration [13] on the other hand provides a platform which can prototype and produce such complex photonic chips with high reliability and low cost.

In this review, we focus on the context of in-door ultra-high-capacity OWC using infrared beams, and discuss the recent development in this area using photonic integration technologies. An overview of photonic integration technologies suitable for such application is given in Section 2. The technologies to achieve chip to free space coupling, which is essential for optical wireless application, is reviewed in Section 3. Then integrated optical wireless receivers and transmitters are discussed in Section 4 and Section 5, respectively. Both basic requirements and recent developments are discussed in details. Finally the review is summarized and the future prospects are given in Section 6 .

\section{Photonic integration technologies}

Photonic integration tends to integrate all the necessary functionalities, including light generation, amplification, modulation, detection, filtering and routing, in a single chip. This is the key to a complete optical system-on-chip, which has huge potential for telecom, datacom, sensing and healthcare applications [13]. Full integration can be achieved either by monolithic approach using one material system (e.g., indium phosphide (InP) [13]), or by heterogeneous/hybrid methods using combined material systems (e.g., $\mathrm{InP} /$ silicon) [14]).

Active/passive integration is one of the most important features for photonic integration technologies used in practical applications such as the OWC. The transmitters and receivers have to be compact and low power consumption, in order to be operated in small, battery-driven and mobile smart devices. Therefore an incomplete chip with external light source is not considered in this context, because of extra assembly cost and optical coupling loss. To achieve on-chip light source and amplification, InP material system is widely used, thanks to its direct bandgap and high quantum efficiency.

Carrying the promise of high performance [15], the monolithic InP platforms have became dominant in the telecom transceiver market. Therefore the InP platforms are also well suited to building integrated transceiver chips for the optical wireless applications. As mentioned earlier, the integrated transceiver chips embedded in smart devices have to be low cost and highly reliable, in order to meet the fast growing 

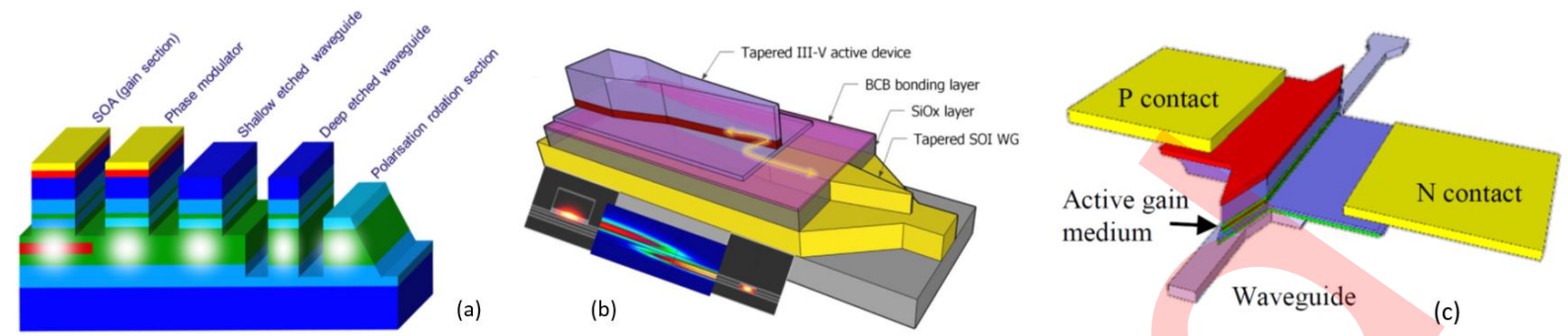

Figure 1 Schematic illustration of typical (a) generic InP platform [13], (b) InP/Si heterogeneous laser/amplifier [22] and (c) InP membrane laser/amplifier [27]. (C) 2018 IOP \& OSA.

number of devices and capacity of data. The rapid and successful development of InP generic integration in recent years $[13,16]$ has enabled revolutions in both cost and reliability. The key ingredients in the generic integration concept are the basic building blocks (BBBs) and the generic process flow [13, 17]. The generic process flow enables a full integration of various functionalities on the same substrate, as illustrated in Fig. 1(a) [13]. The BBBs are standardized components which can be easily assembled into a complex system. They can be fabricated in multi-project wafer (MPW) runs using a single generic process flow. In this case, the costs of $\mathrm{R} \& \mathrm{D}$, material and process can be shared by multiple users and therefore reduced significantly. The reliability is also much improved thanks to the standardization in both process and design.

Silicon photonics has attracted much interest due to its high optical confinement [18]. Sub-micron waveguides and diffractive optics can be realized. Thanks to the CMOS compatibility, the passive circuits (as well as some $\mathrm{Si} / \mathrm{Ge}$ devices) can be fabricated in large wafer scale and volume throughput using existing silicon foundries. However silicon has indirect bandgap. It cannot be used as efficient material for light generation and amplification. To enable on-chip electrical driven sources and amplifiers, heterogeneous integration of InP-based dies are implemented at the desired locations of the silicon photonic circuit. This is done by using adhesive or direct bonding techniques $[19,20]$. Due to the limitation of die-to-wafer bonding, it can be the bottleneck of yield and throughput of heterogeneous $\mathrm{InP} /$ silicon chips. Transfer printing technology has been proposed [21], which has the potential to solve the problem. The footprint of the active components can be also a limiting factor of future higher integration density. Due to the mode mismatch between small silicon waveguides and relatively larger InP mesa, the coupling length between the two has to be very long (in the order of hundreds of $\mu \mathrm{m}$ ), even with optimized multi-stage designs [22]. An example of an InP amplifier section on silicon waveguide is shown in Fig. 1(b) [22].

Another approach with similarly high optical confinement is the InP membranes [23-25]. An example of a twin-guide integration scheme is depicted in Fig. 1(c) [23]. In the InP membrane platforms, the InP functional layerstacks are bonded to a carrier wafer with a low-index optical buffer layer in between. The resulted refractive index contrast is therefore very similar to that of the silicon-on-insulator (SOI) waveguides. An advantage of the $\mathrm{InP}$ membranes is the flexibility of innovation in the active layerstack, to reduce the active/passive coupling length [23] and enhance fill factor in quantum wells (QWs) [24]. Another advantage is the possibility of realizing functional structures on both sides of the membrane [26]. The original surface before bonding can be processed to form topologies up to $2 \mu \mathrm{m}$ [27]. Reliable and uniform bonding quality can still be obtained with such significant surface topologies, using optimized adhesive bonding procedures. This gives huge potential in realizing otherwise incompatible active components (e.g., lasers and uni-travelling carrier (UTC) photodetectors) in the same membrane without additional regrowth steps.

\section{Chip to free space coupling}

The coupling from guided modes in waveguides on chip to the free space and vice versa are crucial for OWC. The coupling loss at the chip to free space interface is crucial for power budget management of the entire link. The far field property of the coupler is another critical parameter. A wide field of view 
Jiao Y, et al. Sci China Inf Sci 4
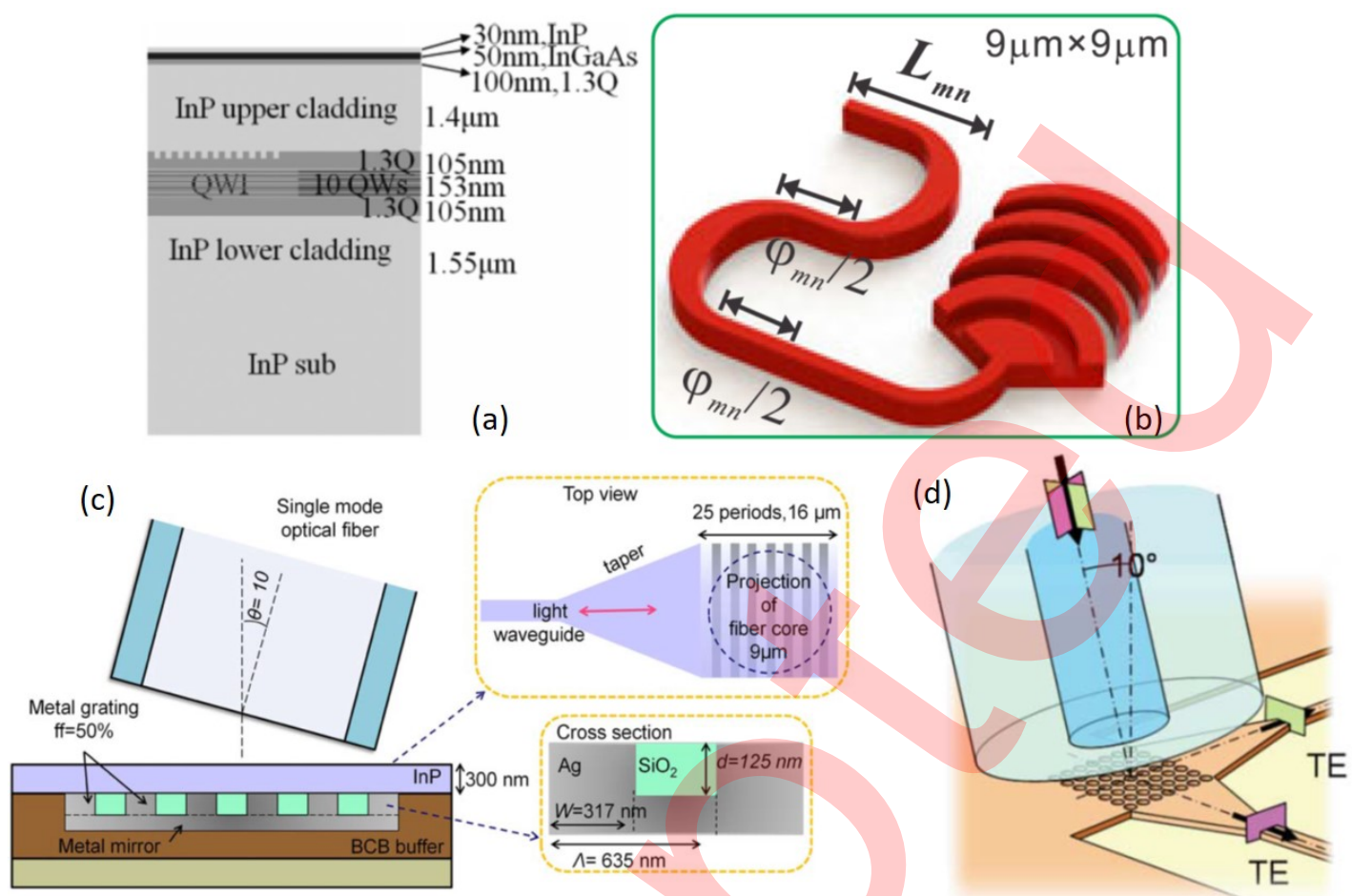

Figure 2 Structures of (a) A weak grating coupler in monothic InP platofmr [28], (b) A compact grating coupler in silicon photonic platform [30], (c) A metallic grating coupler in InP membrane platform using double-side processing technology [36] and a polarization diversity grating coupler [37]. (C) 2018 IEEE \& OSA.

(FOV) is demanded for both transmitters and receivers for optimal beam construction and light collection performances, respectively. The wavelength and polarization sensitivity of the coupler are also important as they determine the tolerance of the system to wavelength shifts and polarization rotation. A wide optical band can further enable wavelength division multiplexing (WDM).

For the monolithic InP platforms, the light is typically emitted and injected through waveguide end facets. Therefore it is only possible to realize 1 dimensional (1D) arrays for beam steering purpose at the edge of the chip. 2D beam steering can be achieved by realizing weak gratings close to the waveguide core, and utilize both phase and wavelength tuning [28]. The waveguide structure is shown in Fig. 2(a) [28]. Since the optical mode in this waveguide system is weakly confined and the interaction with the grating is weak, the full width half maximum (FWHM) of the far field beam profile, which directly relates to the FOV of the free-space coupler, is very limited. The spacing between waveguides in the phased array is another important factor. It determines the number and position of unwanted diffraction orders existing in the far field. A spacing as small as possible with low crosstalk is always preferred. However the waveguide spacing is as large as $5.5 \mu \mathrm{m}$ in [28], due to the limitation of the optical confinement. The resulted steering range is about $16^{\circ}$ within the $+1^{\text {st }}$ and $-1^{\text {st }}$ order peaks. The waveguide spacing can be potentially further optimized using the phase mismatch technique [29].

For the silicon and InP membrane platforms with enhanced optical confinement $[18,23]$, highly efficient surface gratings can be realized to act as the chip to free space couplers. A strongly coupled grating has the advantage of compact footprint. It can diffract most of the light into free space with only a few periods of gratings. An example of a strong grating realized on silicon platform is shown in Fig. 2(b) [30]. The small footprint brings the benefits of significantly wider far field profile (more than an order of magnitude enhancement as compared with [28]), and dense integration of phased arrays. The drawbacks are mainly the loss and the reflection. The strong diffraction will couple significant part of optical power into undesired directions (e.g., downwards into substrate and backwards into input waveguide). The problems can be reduced by using a metal reflector beneath the waveguide to couple the downward light back into the upward direction [31], and using shallower etch depth at the entrance of the 
Jiao Y, et al. Sci China Inf Sci 5

(a)

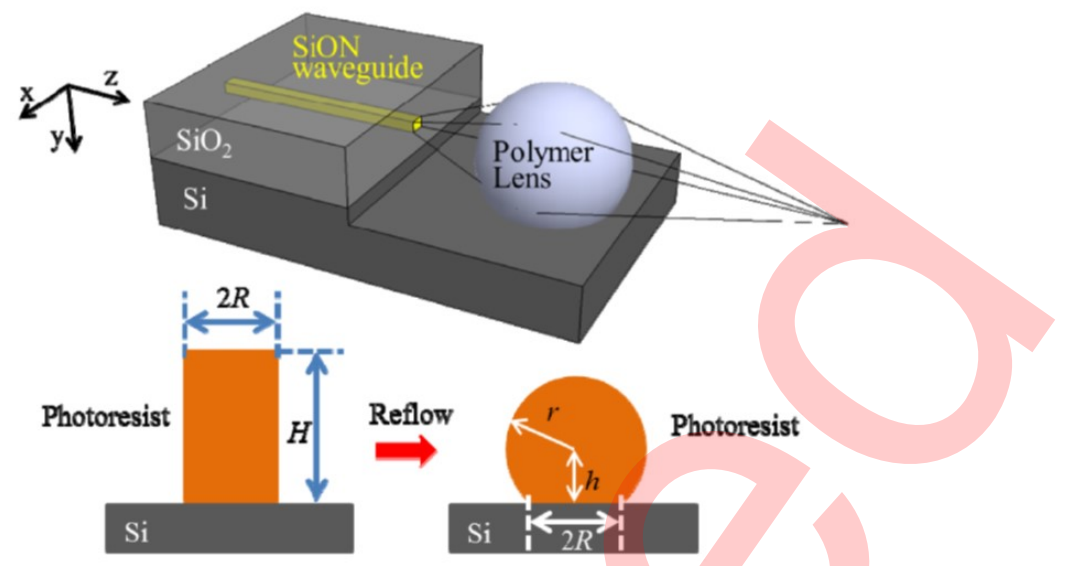

Figure 3 (a) Schematic illustration of polymer-based microlens integrated with waveguides [42] and (b) simplified process mechanism. (C) 2018 OSA.

grating [30] and sub-wavelength structures $[32,33]$ in the grating pitches to reduce back reflection. All these improvements come with the price of increased complexity in technology. Controlling the grating coupling strength in highly confined waveguides is relatively straightforward. One can achieve weakly and strongly coupled gratings by controlling the etch depth of the grating pitches into the waveguide layer. Grating couplers with shallower etch depth (normally half way through the waveguide layer) provide lower loss and reflection, but with a relatively larger footprint $[34,35]$. They have been widely deployed as the interface with optical fibers. Their maturity and ease of design and fabrication make them suitable for low-cost low-standard solutions.

Grating couplers made from metals can provide very high efficiency. For instance one can realize metallic grating structures beneath the waveguide layer, as shown in Fig. 2(c) [36]. The advantage of using metal is that one can create strong light diffraction without the need to etch through waveguide layer. Metals (e.g., silver) with relatively low optical loss are chosen. The metal grating only affect the evanescent field of the optical mode. The optical power stored in the waveguide core will not be disturbed and the loss can be reduced. Strong grating can still be achieved thanks to the significant difference in real part of the refractive indeces between metal and semiconductor/dielectrics. The metal grating proposed in [36] has the promise to achieve efficiency as high as $89 \%$.

Since the gratings rely on the interference of light, they are typically polarization sensitive. This property is important for application in the optical wireless receiver. The light emitted from the light source is typically linearly polarized. The polarization state is uncertain at the receiver side, depending on the optical path and the relative position of the receiver. 2D grating designs $[37,38]$, as shown in Fig. 2(d), can be potentially helpful to this problem. It can guarantee that the light extinction when a particular polarization state is received will not occur. Polarization scrambling [39] to the laser output is another potential technology to solve the problem.

On-chip micro-lenses can be very important to the optical wireless applications, since they can serve as a direct waveguide-to-free-space coupler, or improve the far field property of grating couplers. Microlenses fabricated with cleanroom technologies is preferred in an fully integrated optical system. Polymer based microlenses are promising candidates with good optical properties and ease of fabrication, especially in large arrays. They can be realized by photolithography [40], molding [41] and thermal reflow [42] technologies. The microlens fabricated with reflow technology has been integrated with optical waveguides [42] (schematically shown in Fig.3), showing huge potential to be integrated with surface grating couplers in the future. 
Jiao Y, et al. Sci China Inf Sci 6
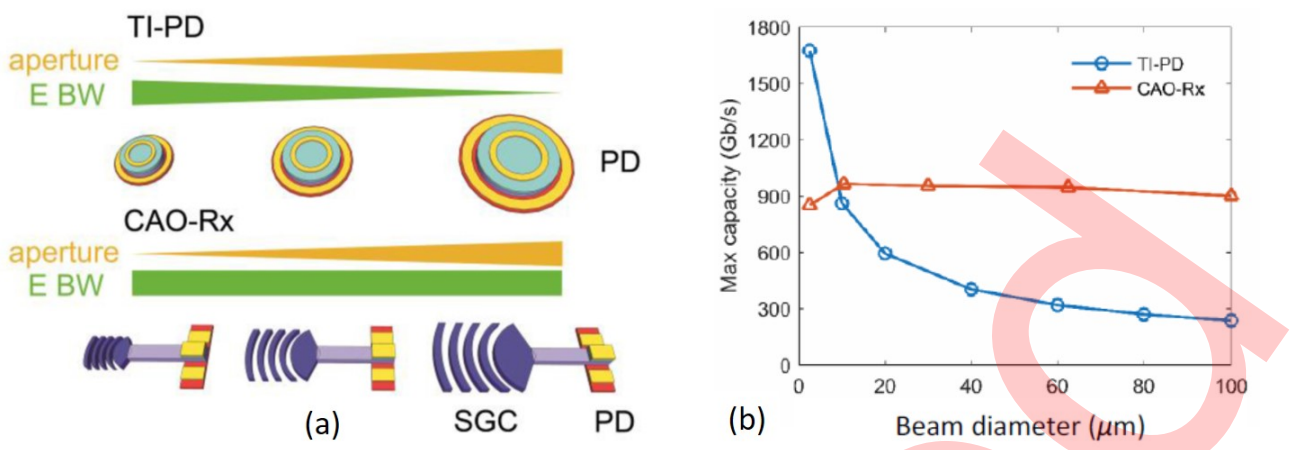

Figure 4 (a) Schematic illustration and (b) maximum predicted capacity of TI-PDs and novel cascaded receivers [11]. (C) 2018 IEEE.

\section{Integrated receivers}

As discussed earlier, the receiver for optical wireless signals needs to be compact and low cost. to meet the drastic increase of number of devices. Therefore the optical coupler, which captures free space light, and the photodetector (PD), which converts optical signal into electrical signal for further processing, need to be integrated in a single chip. The most straightforward way is to use top-illuminated PDs (TI-PDs) [4345], which were commonly used in LED-based VLC systems. For the TI-PDs, a large surface area is preferred to collect free space light efficiently. However this comes with an increased capacitance, which reduces the electrical bandwidth. This optical/electrical tradeoff potentially hinders the development of ultra-high-capacity receivers. Since the TI-PDs directly converts optical signal into electrical domain when having received the light, the information on the phase of the light is lost. Therefore it is not possible to trace back the direction of the incident beam, even using TI-PD arrays.

A new class of optical receivers has been proposed [11,46,47], using the concept of cascaded apertures. The collection of the light is performed by an independent coupler (e.g., a surface grating coupler). Through the coupler, the collected light becomes confined and guided in a waveguide. On the other end of the waveguide, an optimized fast photodetector is placed to convert the light into electrical signal. The coupler can be optimized for a large range of beam diameters, and provide high light collection efficiency without sacrificing the performance of the photodetector. The schematic illustration of the cascaded receivers and the comparison with TI-PDs are depicted in Fig. 4(a). The theoretical maximum data capacities of TI-PD and cascaded receiver are calculated, as shown in Fig. 4(b). The cascaded receiver shows significant advantage over the TI-PD as the beam diameter is larger than $10 \mu \mathrm{m}$.

Both optical coupler and the photodetector have been studied individually. The surface grating coupler was chosen as the optical coupler due to its ease of fabrication and flexibility to various beam diameters. A FOV of about $10^{\circ}$ was demonstrated using a non-optimized surface grating design. The surface grating design used in $[11,46,47]$ can maintain its high coupling efficiency $(>50 \%)$ for a wide range of beam diameters from $1.25 \mu \mathrm{m}$ up to $31.2 \mu \mathrm{m}$. No modification and complication in the fabrication process is needed. Only minor design adaption is needed for the apodization of the grating. It is worth mentioning that, comparing with TI-PDs, the grating couplers are wavelength dependent due to their inteferometric nature. At a fixed acceptance angle, the efficiency drops when the wavelength shifts from the central one. Typical grating couplers have a $3 \mathrm{~dB}$ optical bandwidth ranging from tens of $\mathrm{nm}$ to beyond $100 \mathrm{~nm}$, which is sufficient for OWC application with WDM.

For the photodetector, both p-i-n and UTC schemes have been evaluated and demonstrated. The p-i-n type photodetector is based on reverse bias of a short section of an optical amplifier [46]. It has the advantage of simple design and intrinsic integration with optical sources, which is very valuable for transceivers. The UTC photodetector on the other hand is based on a special layerstack design, which is optimized for speed and linearity [48]. It can have much higher electrical bandwidth and linearity as compared with p-i-n photodetectors. But integration with optical amplifiers will require special considerations [23]. 
Jiao Y, et al. Sci China Inf Sci 7

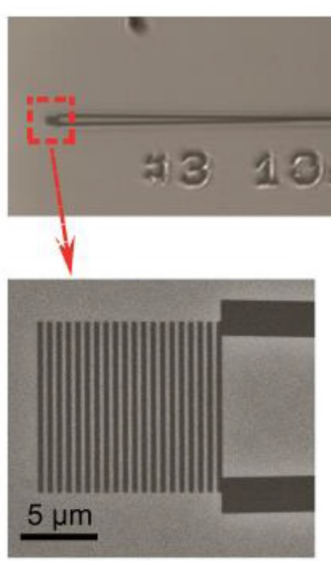

(b)

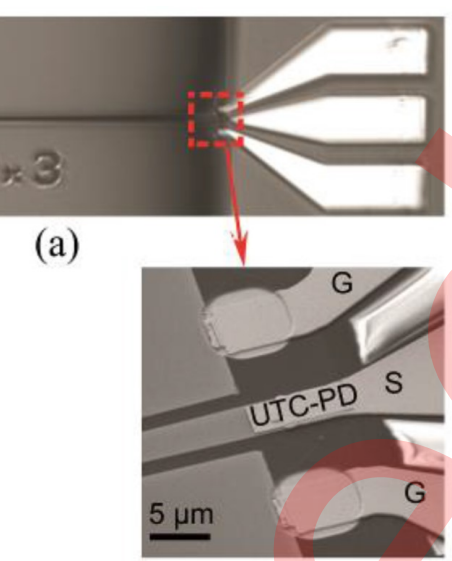

(c)

Figure 5 Pictures of the fabricated cascaded receiver using a UTC photodetector and a surface grating coupler [11,47]. (a) Complete device, (b) surface grating coupler and (c) UTC photodetector. (C) 2018 IEEE.

First demonstrations show promising performance and huge potential for further improvements. Using a single receiver based on a p-i-n photodetector and a surface grating coupler, 17.4 Gbit/s OFDM wireless transmission is obtained [46]. By replacing the p-i-n photodetector with the UTC counterpart, transmission of 5 WDM channels with $40 \mathrm{Gbit} / \mathrm{s}$ OOK signal in each channel has been demonstrated in a single receiver $[11,47]$. The pictures of the fabricated receiver used in $[11,47]$ are shown in Fig. 5 .

The cascaded receiver has been intensively studied in the InP membrane platform [23]. It can be also adapted to the silicon photonic platforms, using silicon-based grating couplers [31] and heterogeneously integrated photodetectors (e.g., based on $\mathrm{Si} / \mathrm{Ge}[49]$ or $\mathrm{InP} / \mathrm{Si}$ [50] integration schemes).

Future work of the novel cascaded receivers can be focused on improvements of the optical aperture. The FOV can be improved by optimizing the coupling strength and footprint of the surface grating, while keeping a balance with the coupling efficiency. The polarization dependency of the surface grating can also be improved by e.g., using the concept of polarization diversity $2 \mathrm{D}$ gratings, as discussed in Section. 3. Furthermore, integration of on-chip wavelength router and integration of receiver arrays with phase control can be studied to realize a fully integrated receiver system-on-chip.

\section{$5 \quad$ Integrated transmitters}

The requirement and implementation of integrated optical wireless transmitters can be different for access points or user terminals. At access point, the key requirements are the room coverage and beam quality. And for users, cost and compactness are of more focus.

To be able to cover the entire room with multiple receivers, the access point must be able to handle multiple beams at the same time. One approach is to use an array of light sources, where each of them is integrated with independent micro-optics. For instance an array of vertical cavity surface emitting lasers (VCSELs) can be integrated with an array of micro-machined lenses [51-53]. In this approach, the number of channels is fundamentally limited by the number of laser/lens pairs integrated in a chip. Therefore the scalability is limited. Another approach is the wavelength-steered pencil beams [7]. A pair of crossed gratings act as the steering element, which can diffract different wavelengths into 2D space. To scale up the number of channels, one just needs to add wavelengths. Those mature grating elements are commercially available and low cost. Moreover they provide high beam quality thanks to the high grating orders used [7]. To assemble a complete transmitter at the access point together with the grating elements, high-performance mass-produced and compact light sources are of high demand. Since wavelength tuning is a critical aspect for the wavelength-steered pencil beams, the quality of single wavelength lasing and the speed of wavelength sweeping are of great importance. The single wavelength lasing typically requires a wavelength filter within the laser cavity. Photonic integration can achieve 
Jiao Y, et al. Sci China Inf Sci 8

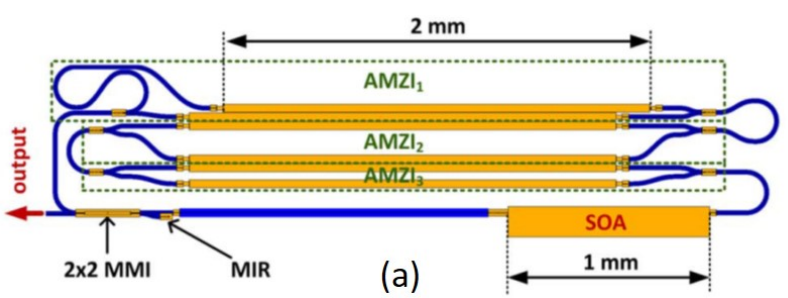

(b)
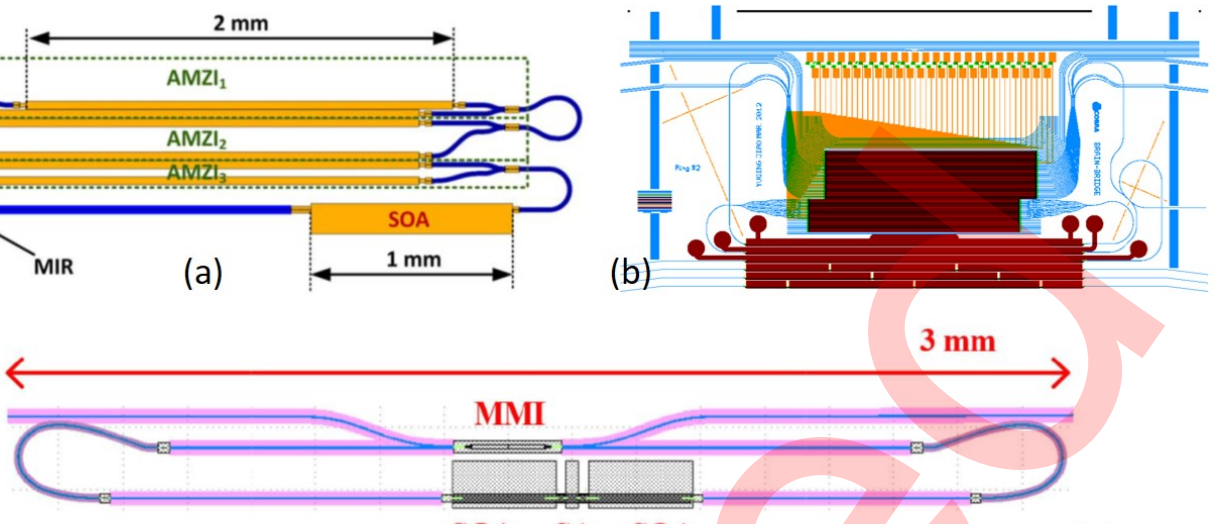

SOA SA SOA

(c)

Figure 6 Pictures of the high-performance lasers realized on generic InP platforms, with (a) record-wide tuning range in a single chip [55] (b) fast wavelength continuous sweep [57,58] and (c) wide optical combs [59].

integrated wavelength filters (e.g., arrayed waveguide gratings (AWGs) [54], Mach-Zehnder inteferometers (MZI) [55] and ring resonators [56] as wavelength selection element in laser cavities. The fast wavelength switch and sweep speed can be ensured by the fast pico-second electro-optic effects [13]. Phase modulators with bandwidth higher than $40 \mathrm{GHz}[13]$ is feasible in the InP photonic integration platforms. Such integrated laser chips can work with free-space grating elements to provide tunable wavelengths or multifrequency combs. High performance lasers realized on commerically available generic InP platforms can achieve record-wide tuning range with a single chip [55], 4000 wavelength continuous fast sweep in just $2 \mathrm{~ms}[57,58]$ as well as monolithically produced extra-wide optical combs [59]. Layout of the above mentioned lasers are depicted in Fig. 6. They are all designed and fabricated using generic BBBs and process flows, showing promising potential to be used in commercialization of the high-performance optical wireless transmitters.

Integrated transmitters at user terminals demand phased-array optical antennas monolithically integrated with light sources, for compactness and low cost. The monolithic InP platforms can realize 1D phased array (shown in Fig. 7(a)) composed of a tunable laser and weak gratings [28]. Full integration of light sources, phase control elements, grating couplers and photodetectors is achieved, thanks to the advantage of $\mathrm{InP}$ photonic integration. Very similar layout has been realized on heterogeneous $\mathrm{InP} / \mathrm{Si}$ chips [60-62]. These chips use phase for steering one axis and wavelength for the other. This requires a tunable laser which typically costs more than a simple single-wavelength laser, due to the complexity and design efforts. The cost of tunable laser is acceptable for the access point as only one or few is needed in a room, but may potentially be a hinder for very low cost user terminals. In this sense, 2D phased array using compact surface gratings is more favorable for the integrated transmitters at user terminal. Many demonstrators based on silicon photonic platforms have been reported [30,63], integrating 2D arrays of grating couplers, phase control elements and photodetectors. An example is shown in Fig. 7(b). The integration of a single-wavelength laser with such systems is not yet available. But it can be achieved in the future using the heterogeneous integration schemes discussed in Section 2. In [64], it is also suggested that plasmonic nanoantennas can be used to replace traditional grating couplers. Those nanoantennas can achieve significant footprint reduction, therefore enables sidelobe-free emission.

Precise phase and amplitude control are critical requirements in the optical phased array. Each phased element (e.g., grating couplers) must be independently controlled in phase and amplitude. The former is the key to the beam steering, and the latter contributes largely to the quality of beam construction. On-chip phase control can be realized with thermal-optic or electro-optic effects on the optical waveguide. Electro-optic effects are fast effects with nanosecond dynamics [65,66]. Therefore it is suitable for fast $2 \mathrm{D}$ surface scan, such as in vehicle light detection and ranging (LiDAR) applications. The footprint of electro-optic phase shifters is relatively large due to the weak electro-optic effects. The length to obtain $2 \pi$ phase shift is typically in the order of hundreds of microns to few millimeters, thus becoming bottleneck 
Figure 7 Layouts of the (a) fully integrated 1D phased array with additional wavelength steering [28], (b) 2D phased array using silicon photonic technology [63].

of the integration density and scalability. Electro-optic polymer is another candidate to provide fast and efficient phase shift $[67,68]$. It has been intensively studied in in context of high-speed optical modulators. The relatively short lifetime and unverified compatibility issue with other components (lasers, optical antennas) may hinder its application described in this review. Thermo-optic effect is usually regarded as a relatively slow effect, with time constants in the order of microsecond to millisecond $[69,70]$. However, for indoor OWC application, the movement speed of human with portable smart devices is in the order of meter per second. Assuming a ceiling height of 3 meters, the required tuning speed of the optical beam from the transmitter is in the order of just $10^{-4} \mathrm{rad} / \mathrm{ms}$. Therefore the response speed of the thermooptically tuned optical beam can easily catch up with such indoor movement. Significant reduction of footprint (as short as about $10 \mu \mathrm{m}$ ) in thermo-optic phase shifters has been reported in CMOS-compatible doped silicon waveguides [30].

A straightforward approach to achieve amplitude control in each phased array element is using a tunable attenuator, such as an MZI integrated with heater [63]. This passive approach brings convenience in design and process, but sacrifices the output power. It is due to the fact that all the other optical antenna elements have to be attenuated to accommodate just one or few elements with higher path losses. Amplitude control with on-chip optical amplifiers would provide full flexibility from attenuation to amplification, simply using either forward or reverse bias. High performance optical amplifiers with the capability to be integrated with high-confinement waveguides and optical antennas are available in several platforms, with the potential of large-scale deployment using either wafer-scale [27] or transferprinting processes [21]. Attention is needed in the near future in the thermal management of high density optical amplifiers, especially in terms of thermal crosstalk in high density circuits [71].

Reconfigurable transceivers can be a promising low-cost solution, which acts as receiver or transmitter using same set of devices. This concept has been proposed in [72], which is schematically shown in Fig. 8. The key element in such concept is a reconfigurable gate, which can be realized by a p-i-n diode. In receiver mode, the p-i-n diode acts as a photodetector and converts collected light from grating coupler into electrical signal. If the length of the diode is designed properly, it can block the light from propagating 
Jiao Y, et al. Sci China Inf Sci 10
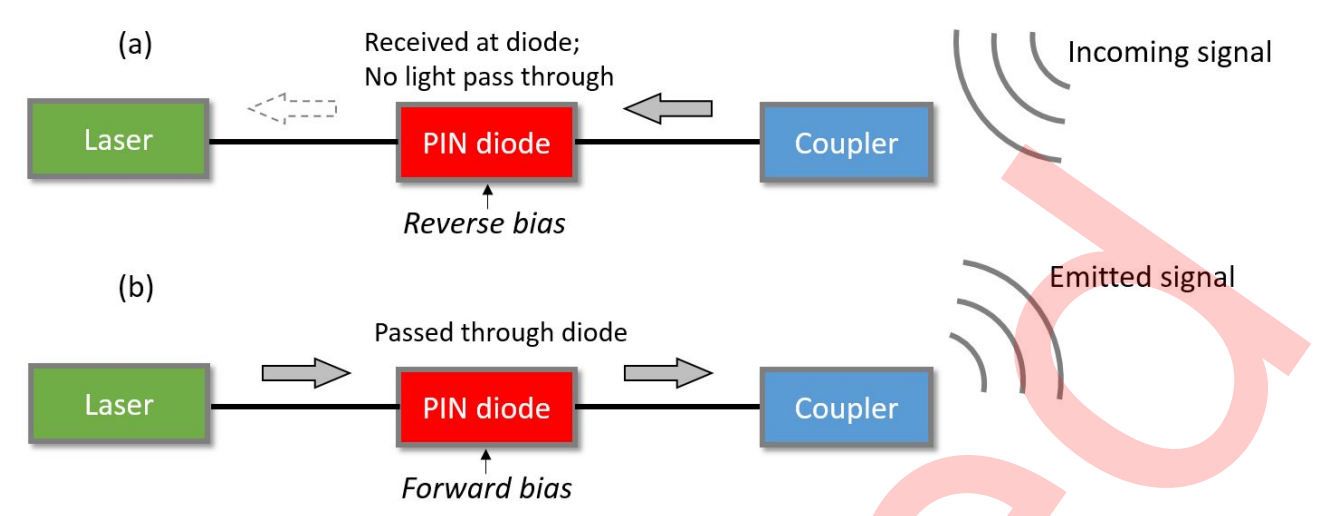

Figure 8 Schematic illustration of the reconfigurable optical wireless transceiver proposed in [72]: (a) receiver mode, (b) transmitter mode.

towards next stage of the circuit. In transmitter mode, the p-i-n diode can be transparent to light when being forward biased to a certain level. Therefore on-chip light source can directly send light through the grating coupler into the free space. Such reconfigurability needs to be operated in divided time, which has potential in applications such as the broadcast and data exchange among wireless devices. Initial demonstrative experiment has been carried out [72]. In the work, a p-i-n diode was integrated with a grating coupler, forming a basic element of the reconfigurable receiver. Due to fabrication issue, the detector has only $2 \mathrm{GHz}$ electrical bandwidth. However data transmission of $17.4 \mathrm{Gbit} / \mathrm{s}$ OFDM signal with 16-QAM modulation was still successful, showing the capability of this simple p-i-n diode in handling complex data formats. Transmitter mode was not reported in the work due to the same issue in the diodes. With the development of high-performance active diodes [27], the proposed reconfigurable scheme has a high promise in the near future.

\section{$6 \quad$ Summary and prospects}

In this paper, the recent development of infrared-beam OWC using photonic integrated technology is reviewed. The rapid maturing of photonic integration can provide high-performance, low-cost and compact solutions for optical wireless signal transmission in the indoor scenario. Tens of Gbit/s data transmission has been achieved in a single device level. On the other hand, high integration density up to thousands of components per chip can boost the chip complexity and performance towards fully integrated OWC system-on-chip.

The optical wireless chips can also co-integrate with microwave photonic functionalities, as discussed in [73]. The radio beams have larger beam spread, hence wider coverage, while the optical beams are concentrated, hence secure and high-capacity. Since they share a lot of basic functional components, the integrated optical wirless systems and microwave systems have the potential to be constructed in the same platform using same technology and BBBs.

Electronics is indispensable in any optical communication systems. The co-integration of photonics and electronics can result in significant reduction of packaging costs, parasitics as well as power consumptions. It is therefore very promising for future OWC. A silicon photonics based demonstrator is reported in [74], which integrated photonic interconnects in a microprocessor, yet with the absence of on-chip light source. Another promising concept is the wafer-scale integration of photonic and electronic circuits in heterogeneous layers with very short interconnections [23]. In this approach, possible incompatibilities of technologies used for photonics and electronics can be minimized, and the mature design libraries and process technologies from both worlds can be utilized.

Finally, the bright future of integrated OWC chips is enabled by successful commercialization and openaccess of photonic integration platforms [75-79]. A pulling driving force from the industry is foreseen. Prototyping with reduced cost through MPW runs will attract significant industrial designers, especially 
the small and medium-sized enterprises (SMEs).

Acknowledgements This work was supported by NWO Gravitation project Integrated Nanophotonics, and ERC Advanced Grant projects NOLIMITS and BROWSE.

Conflict of interest The authors declare that they have no conflict of interest.

\section{References}

1 W. Ejaz, A. Anpalagan, M.A. Imran, M. Jo, M. Naeem, S.B. Qaisar, W. Wang. Internet of Things (IoT) in 5G Wireless Communications. IEEE Access, 2016, 4:10310-10314.

2 Q.C. Li, H. Niu, A.T. Papathanassiou, G. Wu. 5G Network Capacity: Key Elements and Technologies. IEEE Vehicular Technology Magazine, 2014, 9:71-78.

3 A.M.J. Koonen, E. Tangdiongga. Photonic Home Area Networks. Journal of Lightwave Technology, 2014, 32:591-604.

4 X. Li, R. Lu, X. Liang, X. Shen, J. Chen, X. Lin. Smart community: an internet of things application. IEEE Communications Magazine, 2011, 49:68-75.

5 S.M.R. Islam, D. Kwak, M.H. Kabir, M. Hossain, K.S. Kwak. The Internet of Things for Health Care: A Comprehensive Survey. IEEE Access, 2015, 3:678-708.

6 F. Boccardi, R.W. Heath, A. Lozano, T.L. Marzetta, P. Popovski. Five disruptive technology directions for 5G. IEEE Communications Magazine, 2014, 52:74-80.

7 T. Koonen, J. Oh, K. Mekonnen, Z. Cao, E. Tangdiongga. Ultra-High Capacity Indoor Optical Wireless Communication Using 2D-Steered Pencil Beams. Journal of Lightwave Technology, 2016, 34:4802-4809.

8 V. Jungnickel, D. Schulz, J. Hilt, et al. Optical wireless communication for backhaul and access. In: 2015 European Conference on Optical Communication (ECOC), Valencia, Spain, 2015. 0643.

9 D. O'Brien, R. Turnbull, H. Minh, et al. High-Speed Optical Wireless Demonstrators: Conclusions and Future Directions. Journal of Lightwave Technology, 2012, 30:2181-2187.

10 H. Haas. Visible Light Communication. In: Optical Fiber Communication Conference, Los Angeles, CA, USA, 2015. Tu2G.5.

11 Z. Cao, Y. Jiao, L. Shen, X. Zhao, R. Stabile, J.J.G.M. van der Tol, T. Koonen. Ultra-high Throughput Indoor Infrared Wireless Communication System Enabled by a Cascaded Aperture Optical Receiver Fabricated on InP Membrane. Journal of Lightwave Technology, 2018, 36:57-67.

12 J. Summers, T. Vallaitis, P. Evans, et al. 40 Channels x 57 Gb/s monolithically integrated InP-based coherent photonic transmitter. In: 2014 European Conference on Optical Communication (ECOC), Cannes, France, 2014. P.2.5.

13 M. Smit, X. Leijtens, H. Ambrosius, et al. An introduction to InP-based generic integration technology. Semiconductor Science and Technology, 2014, 29:083001.

14 G. Roelkens, A. Abassi, P. Cardile, et al. III-V-on-Silicon Photonic Devices for Optical Communication and Sensing. Photonics, 2015, 2:969.

15 L.A. Coldren, S.C. Nicholes, L. Johansson, et al. High performance InP-based photonic ICs a tutorial. Journal of Lightwave Technology, 2011, 29:554-570.

16 K.A. Williams, E.A.J.M. Bente, D. Heiss, et al. InP photonic circuits using generic integration [Invited]. Photonics Research, 2015, 3:B60-B68.

17 M. Smit, X. Leijtens, E. Bente, et al. Generic foundry model for InP-based photonics. IET Optoelectronics, 2011, $5: 187-194$

18 W. Bogaerts, R. Baets, P. Dumon, et al. Nanophotonic waveguides in silicon-on-insulator fabricated with CMOS technology. Journal of Lightwave Technology, 2005, 23:401-412.

19 G. Roelkens, J. Van Campenhout, J. Brouckaert, et al. III-V/Si photonics by die-to-wafer bonding. Materials Today, 2007, 10:36-43.

20 D. Liang, G. Roelkens, R. Baets, J. Bowers. Hybrid Integrated Platforms for Silicon Photonics. Materials, 2010, 3:1782-1802.

21 X. Luo, Y. Cheng, J. Song, et al. Wafer-Scale Dies-Transfer Bonding Technology for Hybrid III/V-on-Silicon Photonic Integrated Circuit Application. IEEE Journal of Selected Topics in Quantum Electronics, 2016, 22:443-454.

22 S. Keyvaninia, S. Verstuyft, L. Van Landschoot, et al. Heterogeneously integrated III-V/silicon distributed feedback lasers. Optics Letters, 2013, 38:5434-5437.

23 J.J.G.M. van der Tol, Y. Jiao, L. Shen, et al. Indium Phosphide Integrated Photonics in Membranes. IEEE Journal of Selected Topics in Quantum Electronics, 2018, 24:6100809.

24 D. Inoue, T. Hiratani, K. Fukuda, et al. Integrated Optical Link on Si Substrate Using Membrane Distributed-Feedback Laser and p-i-n Photodiode. IEEE Journal of Selected Topics in Quantum Electronics, 2017, 23:3700208.

25 S. Matsuo, T. Fujii, K. Hasebe, et al. Directly modulated buried heterostructure DFB laser on $\mathrm{SiO}_{2} / \mathrm{Si}_{\mathrm{substrate}}$ fabricated by regrowth of InP using bonded active layer. Optics Express, 2014, 22:12139-12147.

26 L. Shen, Y. Jiao, A. Higuera Rodriguez, et al. Double-sided processing for membrane-based photonic integration. In: 18th European Conference on Integrated Optics (ECIO 2016), Warsaw, Poland, 2016.

27 V. Pogoretskiy, J. van Engelen, J. van der Tol, et al. An Integrated SOA-building block for an InP-membrane platform. In: Advanced Photonics 2017 (IPR, NOMA, Sensors, Networks, SPPCom, PS), New Orleans, USA, 2017. Postdeadline Downloaded to IP: 131.155.212.127 On: 2018-03-29 18:18:57 http://engine.scichina.com/doi/10.1007/s11432-018-9391-1 
Jiao Y, et al. Sci China Inf Sci 12

paper JW4A.1.

28 W. Guo, P.R.A. Binetti, C. Althouse, et al. Two-Dimensional Optical Beam Steering With InP-Based Photonic Integrated Circuits. IEEE Journal of Selected Topics in Quantum Electronics, 2013, 19:6100212.

29 W. Song, R. Gatdula, S. Abbaslou, et al. High-density waveguide superlattices with low crosstalk. Nature Communications, 2015, 6:7027.

30 S. Jie, E. Timurdogan, A. Yaacobi, et al. Large-Scale Silicon Photonic Circuits for Optical Phased Arrays. IEEE Journal of Selected Topics in Quantum Electronics, 2014, 20:264-278.

31 F. Van Laere, G. Roelkens, M. Ayre, et al. Compact and highly efficient grating couplers between optical fiber and nanophotonic waveguides. Journal of Lightwave Technology, 2007, 25:151-156.

32 Y. Wang, X. Wang, J. Flueckiger, et al. Focusing sub-wavelength grating couplers with low back reflections for rapid prototyping of silicon photonic circuits. Optics Express, 2014, 22, 20652-20662.

33 A.J. Millan-Mejia, Y. Jiao, J.J.G.M. van der Tol, M.K. Smit. Design of an optical nanoantenna with focusing subwavelength grating couplers and metallic reflector. In: 24th Optical Wave And Waveguide Theory And Numerical Modelling Workshop (OWTNM 2016), Warsaw, Poland, 2016.

34 D. Taillaert, W. Bogaerts, P. Bienstman, et al. An out-of-plane grating coupler for efficient butt-coupling between compact planar waveguides and single-mode fibers. IEEE Journal of Quantum Electronics, 2002, 38:949-955.

35 Y. Jiao, J. Pello, A. Millan Mejia, et al. Fullerene-assisted electron-beam lithography for pattern improvement and loss reduction in InP membrane waveguide devices. Optics Letters, 2014, 39:1645-1648.

36 A. Higuera-Rodriguez, V. Dolores-Calzadilla, Y. Jiao, et al. Realization of efficient metal grating couplers for membrane-based integrated photonics. Optics Letters, 2015, 40:2755-2757.

37 F. Van Laere, T. Stomeo, D. Taillaert, et al. Efficient Polarization Diversity Grating Couplers in Bonded InPMembrane. IEEE Photonics Technology Letters, 2008, 20:318-320.

38 M. Streshinsky, R. Shi, A. Novack, et al. A compact bi-wavelength polarization splitting grating coupler fabricated in a $220 \mathrm{~nm}$ SOI platform. Optics Express, 2013, 21:31019-31028.

39 F. Heismann, R.W. Smith. High-speed polarization scrambler with adjustable phase chirp. IEEE Journal of Selected Topics in Quantum Electronics, 1996, 2:311-318.

40 R. Yang, W. Wang. Out-of-plane polymer refractive microlens fabricated based on direct lithography of SU-8. Sensors and Actuators A: Physical, 2004, 113:71-77.

41 K. Ju-Nan, H. Chia-Chun, Y. Sung-Yi, L. Gwo-Bin. An SU-8 microlens array fabricated by soft replica molding for cell counting applications. Journal of Micromechanics and Microengineering, 2007, 17:693.

42 L. Chang, M. Dijkstra, N. Ismail, et al. Waveguide-coupled micro-ball lens array suitable for mass fabrication. Optics Express, 2015, 23:22414-22423.

43 D.C. O'Brien, G.E. Faulkner, E.B. Zyambo, et al. Integrated transceivers for optical wireless communications. IEEE Journal of Selected Topics in Quantum Electronics, 2005, 11:173-183.

44 H.L. Minh, D. O'Brien, G. Faulkner, et al. A 1.25-Gb/s Indoor Cellular Optical Wireless Communications Demonstrator. IEEE Photonics Technology Letters, 2010, 22:1598-1600.

45 G. Cossu, A.M. Khalid, Choudhury P, et al. 3.4 Gbit/s visible optical wireless transmission based on RGB LED. Optics Express, 2012, 20:B501-B506.

46 Z. Cao, Y. Jiao, L. Shen, et al. Optical wireless data transfer enabled by a cascaded acceptance optical receiver fabricated in an InP membrane platform. In: Optical Fiber Communication Conference (OFC), Anaheim, California, USA, 2016. M2B.3.

47 Z. Cao, L. Shen, Y. Jiao, et al. 200 Gbps OOK Transmission over an Indoor Optical Wireless Link Enabled by an Integrated Cascaded Aperture Optical Receiver. In: Optical Fiber Communication Conference (OFC), Los Angeles, California, United States, 2017. Postdeadline Papers Th5A.6.

48 L. Shen, Y. Jiao, W. Yao, et al. High-bandwidth uni-traveling carrier waveguide photodetector on an InP-membraneon-silicon platform. Optics Express, 2016, 24:8290-8301.

49 H. Chen, M. Galili, P. Verheyen, et al. 100 Gbps RZ Data Reception in 67 GHz Si-Contacted Germanium Waveguide p-i-n Photodetectors. Journal of Lightwave Technology, 2016, 35:722-726.

50 X. Xie, Q. Zhou, E. Norberg, et al. Heterogeneously Integrated Waveguide-Coupled Photodiodes on SOI with $12 \mathrm{dBm}$ Output Power at $40 \mathrm{GHz}$. Optical Fiber Communication Conference (OFC), Los Angeles, California, United States, 2015. Postdeadline Papers Th5B.7.

51 S.S. Lee, L.Y. Lin, K.S.J. Pister, et al. Passively aligned hybrid integration of $8 \times 1$ micromachined micro-Fresnel lens arrays and $8 \times 1$ vertical-cavity surface-emitting laser arrays for free-space optical interconnect. IEEE Photonics Technology Letters, 1995, 7:1031-1033.

52 E.M. Strzelecka, D.A. Louderback, B.J. Thibeault, et al. Parallel free-space optical interconnect based on arrays ofvertical-cavity lasers and detectors with monolithic microlenses. Applied Optics, 1998, 37:2811-2821.

53 A. Tuantranont, V.M. Bright, J. Zhang, et al. Optical beam steering using MEMS-controllable microlens array. Sensors and Actuators A: Physical, 2001, 91:363-372.

54 B.W. Tilma, Y. Jiao, P.J. van Veldhoven, et al. InP-based monolithically integrated tunable wavelength filters in the 1.6C1.8m wavelength region for tunable laser purposes. Journal of Lightwave Technology, 2011, 29:2818-2830.

55 S. Latkowski, A. Hansel, N. Bhattacharya, et al. Novel Widely Tunable Monolithically Integrated Laser Source. IEEE Photonics Journal, 2015, 7:1-9.

56 T. Komljenovic, J.E. Bowers. Monolithically Integrated High-Q Rings for Narrow Linewidth Widely Tunable Lasers. IEEE Journal of Quantum Electronics, 2015, 51:1-10. 
Jiao Y, et al. Sci China Inf Sci 13

57 B.W. Tilma, Y. Jiao, J. Kotani, et al. Integrated Tunable Quantum-Dot Laser for Optical Coherence Tomography in the $1.7 \mu \mathrm{m}$ Wavelength Region. IEEE Journal of Quantum Electronics, 2012, 48:87-98.

58 Y. Jiao. Towards a monolithically integrated swept-source optical coherence tomography system in the $1.7 \mu \mathrm{m}$ wavelength region. PhD Thesis, Eindhoven University of Technology, 2013, Eindhoven, The Netherlands.

59 V. Moskalenko, J. Koelemeij, K. Williams, E. Bente. Study of extra wide coherent optical combs generated by a QW-based integrated passively mode-locked ring laser. Optics Letters, 2017, 42:1428-1431.

60 J.K. Doylend, M.J.R. Heck, J.T. Bovington, et al. Two-dimensional free-space beam steering with an optical phased array on silicon-on-insulator. Optics Express, 2011, 19:21595-21604.

61 J.C. Hulme, J.K. Doylend, M.J.R. Heck, et al. Fully integrated hybrid silicon free-space beam steering source with 32-channel phased array. In: Proc. SPIE 8989, Smart Photonic and Optoelectronic Integrated Circuits XVI, 2014, 8989:898907-898907-15.

62 D.N. Hutchison, J. Sun, J.K. Doylend, et al. High-resolution aliasing-free optical beam steering. Optica, 2016, 3:887890.

63 H. Abediasl, H. Hashemi. Monolithic optical phased-array transceiver in a standard SOI CMOS process. Optics Express, 2015, 23:6509-6519.

64 M.J.R. Heck. Highly integrated optical phased arrays: photonic integrated circuits for optical beam shaping and beam steering. Nanophotonics, 2016, 6:93-107.

65 J.F. Vinchant, J.A. Cavailles, M. Erman, et al. InP/GaInAsP guided-wave phase modulators based on carrier-induced effects: theory and experiment. Journal of Lightwave Technology, 1992, 10:63-70.

66 K. Liu, C.R. Ye, S. Khan, et al. Review and perspective on ultrafast wavelength-size electro-optic modulators. Laser \& Photonics Reviews, 2015, 9:172C194.

67 J.H. Wlbern, S. Prorok, J. Hampe, et al. $40 \mathrm{GHz}$ electro-optic modulation in hybrid siliconCorganic slotted photonic crystal waveguides. Optics Letters, 2010, 35:2753-2755.

68 R. Palmer, L. Alloatti, D. Korn, et al. Silicon-Organic Hybrid MZI Modulator Generating OOK, BPSK and 8-ASK Signals for Up to $84 \mathrm{Gbit} / \mathrm{s}$. IEEE Photonics Journal, 2013, 5:6600907-6600907.

69 M.W. Pruessner, T.H. Stievater, M.S. Ferraro, W.S. Rabinovich. Thermo-optic tuning and switching in SOI waveguide Fabry-Perot microcavities. Optics Express, 2007, 15:7557-7563.

70 P. Sun, R.M. Reano. Submilliwatt thermo-optic switches using free-standing silicon-on-insulator strip waveguides. Optics Express, 2010, 18:8406-8411.

71 G. Gilardi, W. Yao, H. R. Haghighi, et al. Deep Trenches for Thermal Crosstalk Reduction in InP-Based Photonic Integrated Circuits. Journal of Lightwave Technology, 2014, 32:4864-4870.

72 Y. Jiao, Z. Cao, L. Shen, et al. Membrane-Based Receiver/Transmitter for Reconfigurable Optical Wireless BeamSteering Systems. IEEE Journal of Selected Topics in Quantum Electronics, 2018, 24:6100506.

73 Z. Cao, Q. Ma, A.B. Smolders, et al. Advanced Integration Techniques on Broadband Millimeter-wave Beam Steering for $5 \mathrm{G}$ and Beyond. IEEE Journal of Quantum Electronics, 2016, 52:1-20.

74 C. Sun, M.T. Wade, Y. Lee, et al. Single-chip microprocessor that communicates directly using light. Nature, 2015, 528:534-538.

75 JePPIX. Url http://www.jeppix.eu/

76 Smartphotonics. Url http://www.smartphotonics.nl/

77 Fraunhofer Heinrich Hertz Institute. Url http://www.hhi.fraunhofer.de/

78 Europractice. Url http://www.europractice-ic.com/

79 Photon Delta. Url http://www.photondelta.eu/ 